Quasiparticle Freeze-Out in

Superconducting Tunnel Junction X-Ray

Detectors with Killed Base Electrode

V. A. Andrianov, L. V. Filippenko, S. Friedrich

July 24,2013

15th International Workshop on Low Temperature Detectors (LTD-15)

Pasadena, CA, United States

June 24, 2013 through June 28, 2013 
This document was prepared as an account of work sponsored by an agency of the United States government. Neither the United States government nor Lawrence Livermore National Security, LLC, nor any of their employees makes any warranty, expressed or implied, or assumes any legal liability or responsibility for the accuracy, completeness, or usefulness of any information, apparatus, product, or process disclosed, or represents that its use would not infringe privately owned rights. Reference herein to any specific commercial product, process, or service by trade name, trademark, manufacturer, or otherwise does not necessarily constitute or imply its endorsement, recommendation, or favoring by the United States government or Lawrence Livermore National Security, LLC. The views and opinions of authors expressed herein do not necessarily state or reflect those of the United States government or Lawrence Livermore National Security, LLC, and shall not be used for advertising or product endorsement purposes. 


\title{
Quasiparticle Freeze-Out in Superconducting Tunnel Junction X-Ray Detectors with Killed Base Electrode
}

\author{
V. A. Andrianov ${ }^{1}$, L. V. Filippenko ${ }^{2}$, S. Friedrich ${ }^{3}$ \\ ${ }^{1}$ Lomonosov Moscow State University, Skobeltsyn Institute of Nuclear Physics, 119991 \\ Moscow, Russian Federation \\ ${ }^{2}$ Institute of Radio Engineering and Electronics RAS, 103907 Moscow, Russian Federation \\ ${ }^{3}$ Lawrence Livermore National Laboratory, Livermore, CA 94550, USA
}

\begin{abstract}
The current-voltage characteristics of superconducting tunnel junction (STJ) X-ray detectors were measured in the temperature range from 4.2 to $0.1 \mathrm{~K}$. The freeze-out of the thermal tunneling current was compared between an STJ detector with a traditional $\mathrm{Nb} / \mathrm{Al} / \mathrm{Al}_{2} \mathrm{O}_{3} / \mathrm{Al} / \mathrm{Nb}$ layer structure and a $\mathrm{Ti} / \mathrm{Nb} / \mathrm{Al} / \mathrm{Al}_{2} \mathrm{O}_{3} / \mathrm{Al} / \mathrm{Nb} / \mathrm{NbN}$ detector whose lowgap Ti film kills the X-ray response of the base electrode. The current decrease and the linear low-temperature I(V) characteristics for the detector with the killed electrode can be explained by current contributions from the subgap states of the Ti film. The data are analyzed on the basis of the proximity theory in the dirty limit.
\end{abstract}

Keywords: X-rays detectors $\cdot$ Superconducting tunnel junctions $\cdot$ Quasiparticles $\cdot$ Proximity theory $\cdot$ Density of states

\section{Introduction}

Superconducting tunnel junctions (STJs) are being developed as single photon detectors because of their excellent energy resolution, high speed and low energy threshold of registration [1]. At $5.9 \mathrm{keV}$, their energy resolution of $\sim 10-20 \mathrm{eV}$ is substantially better than the $140-150 \mathrm{eV}$ resolution of typical silicon detectors. In the optical range, they offer photoncounting capability with an energy resolution of about $10 \%$. STJ detectors typically employ multilayer electrodes of superconductors with different transition temperatures $T_{c}$ and different superconducting gaps $\Delta$ to increase the tunneling probability for excess quasiparticles and enable charge multiplication by backtunneling [2]. Traditionally, $\mathrm{Nb} / \mathrm{Al}$ 
bilayer electrodes have been used to form symmetric $\mathrm{Nb} / \mathrm{Al} / \mathrm{AlO}_{\mathrm{x}} / \mathrm{Al} / \mathrm{Nb} \mathrm{STJ}$ detectors, with the thick Nb-layer $\left(\mathrm{T}_{\mathrm{c}} \approx 9 \mathrm{~K}\right)$ serving as the X-ray absorber and the thin Al-layer $\left(\mathrm{T}_{\mathrm{c}} \approx 1.2 \mathrm{~K}\right)$ near the tunnel barrier as a quasiparticle trap. These devices have achieved high energy resolution and higher speed than most other cryogenic detectors, but suffer from a line splitting artifact if photons are absorbed in both electrodes.

An alternative STJ detector design uses a low-gap film to suppress ("kill”) the X-ray response of the STJ base electrode $[3,4]$. We have implemented this design by depositing a low-gap Ti trapping layer $\left(\mathrm{T}_{\mathrm{c}} \approx 0.06 \mathrm{~K}\right)$ below the $\mathrm{Nb} / \mathrm{Al}$ base electrode to ensure quick capture of the excess quasiparticles created by X-ray absorption in the base $\mathrm{Nb}$ film. In addition, we have deposited a $\mathrm{NbN}$ reflection layer on top of the $\mathrm{Nb} / \mathrm{Al}$ absorber electrode to prevent $\mathrm{Nb}$ oxidation and the associated quasiparticle loss at the STJ surface. This detector type not only removes the line splitting artifact, but also speeds up the signal response to $\sim 0.2 \mu$ s [4].

We have investigated the effect of the low-gap Ti base layer on the superconducting gap and the density of states in the base electrode. For this we have compared the temperature changes of the current-voltage characteristics for STJ detectors with and without the Ti base layer. We find that the Ti film affects the density of states throughout the base electrode, and discuss this on the basis of the proximity theory in the dirty limit. This causes the subgap current to decrease more slowly at low temperatures than expected from the BCS theory, and may affect the electronic noise in STJ X-ray detectors.

\section{Experiment}

The STJ detectors were fabricated by magnetron sputtering on a Si-substrate with a buffer layer of amorphous $\mathrm{AlO}_{\mathrm{x}}$. Five rhombus-shaped STJ detectors with a 2:1 ratio of the diagonals and areas $\mathrm{S}=400,400,1600,6400$, and $20000 \mu^{2}$ were arranged on one chip. The films had the polycrystalline structure with grains sizes of $\sim 10 \mathrm{~nm}$. The tunnel junctions had a normal resistivity of $\mathrm{R}_{\mathrm{N}} \mathrm{S} \approx 400 \Omega \mu \mathrm{m}^{2}$. Current leads made from $\mathrm{Nb}$ were attached to the larger angles of rhombus and had the width of 5-10 $\mu \mathrm{m}$. Details of detector preparation are given in [4].

Two types of STJ detectors were prepared: A-type detectors had the traditional $\mathrm{Nb}(200) / \mathrm{Al}(4) / \mathrm{AlO}_{\mathrm{x}} / \mathrm{Al}(10) / \mathrm{Nb}(120 \mathrm{~nm})$ structure, with thicknesses in $\mathrm{nm}$ starting at the substrate. B-type detectors had an additional Ti-layer below and a $\mathrm{NbN}$ passivation layer on top to form $\mathrm{Ti}(30) / \mathrm{Nb}(150) / \mathrm{Al}(4), \mathrm{AlO}_{\mathrm{x}} / \mathrm{Al}(12) / \mathrm{Nb}(200) / \mathrm{NbN}(30 \mathrm{~nm})$. 
I(V) characteristics of A- and B-type STJs were measured in the temperature range from 4.2 to $1.25 \mathrm{~K}$ in a pumped-helium cryostat at the Lomonosov Moscow State University, using a preamplifier with a dc current bias. I(V) curves of B-type STJs were measured at $\mathrm{T}=$ $0.1 \mathrm{~K}$ in an adiabatic demagnetization refrigerator at Lawrence Livermore National Laboratory, using a preamplifier with a dc voltage bias. In each case, the dc Josephson current was suppressed by an external magnetic field along the short diagonal of the devices.

\section{Results and Discussion}

Figures 1 and 2 show the quasiparticle branch of the I(V) curves at different temperatures for A- and B-type STJs. At $4.2 \mathrm{~K}$ the I(V) curves exhibit typical hysteresis, caused by the gap difference in the base and the top electrodes. For A-type STJs, $\Delta_{\text {base }}=1.33$ $\mathrm{mV}, \Delta_{\text {top }}=1.0 \mathrm{mV}$, and $\Delta_{\text {base }}-\Delta_{\text {top }}=0.33 \mathrm{meV}$; and for B-type STJs with the Ti base layer $\Delta_{\text {base }}=1.34 \mathrm{mV}, \Delta_{\text {top }}=0.96 \mathrm{mV}$ and $\Delta_{\text {base }}-\Delta_{\text {top }}=0.38 \mathrm{meV}$. The $\mathrm{Nb}$ gap is slightly reduced form its bulk value due to the proximity effect with the Al films [2, 5], and no effect of the Ti
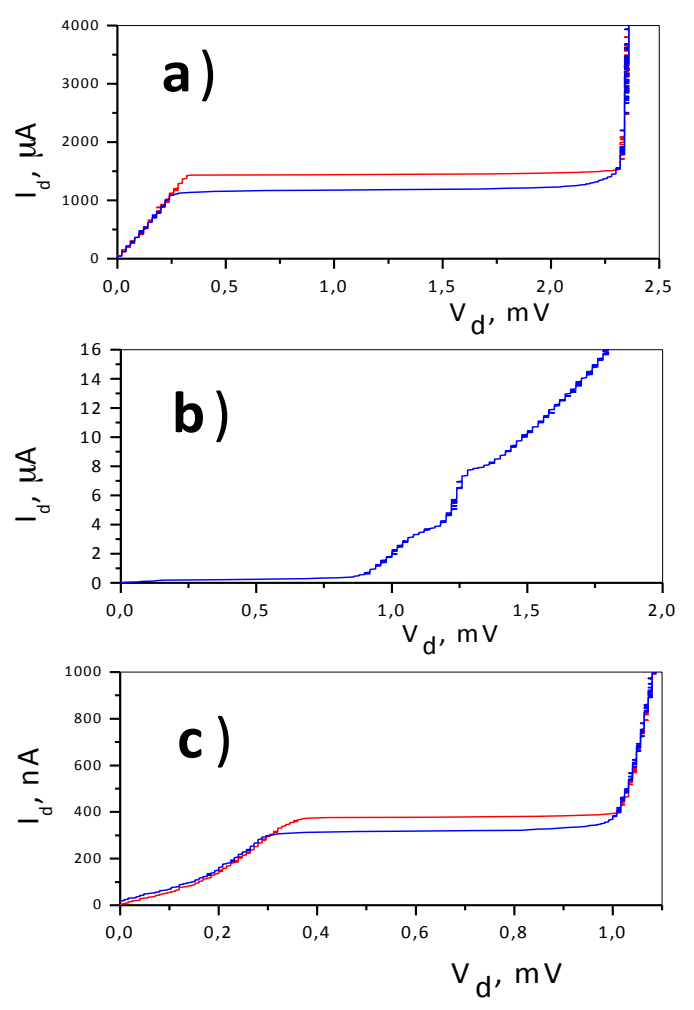

Fig. 1 (Color online) I(V) characteristics of A-type $\mathrm{Nb} / \mathrm{Al} / \mathrm{AlO}_{\mathrm{x}} / \mathrm{Al} / \mathrm{Nb} \mathrm{STJ}$ at $4.2 \mathrm{~K}$

(a) and $1.25 \mathrm{~K}$ (b and c)
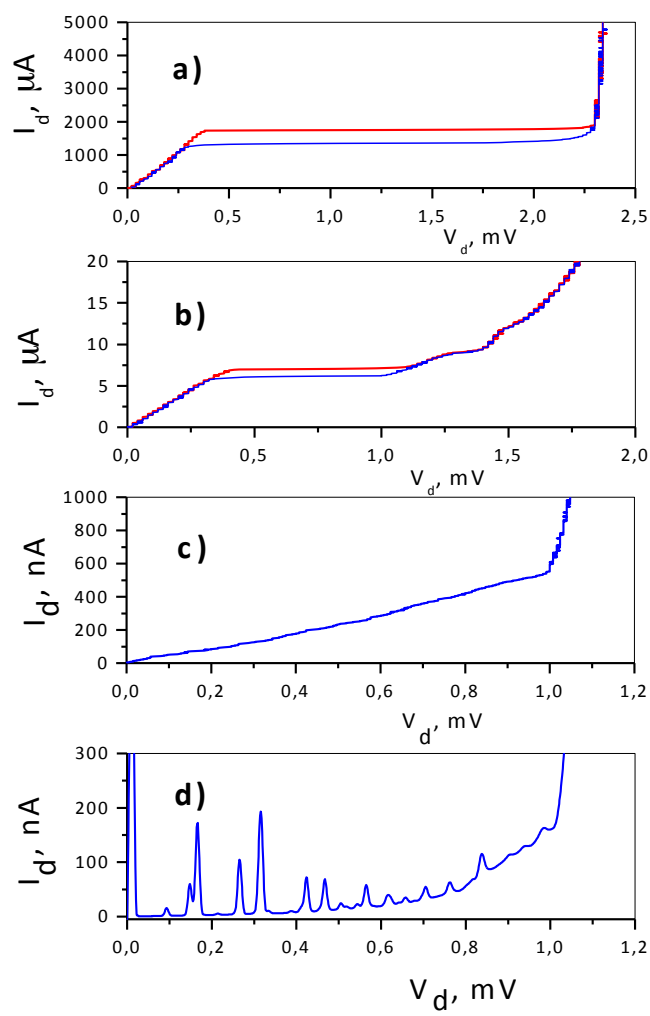

Fig. 2 (Color online) I(V) characteristics of B-type $\mathrm{Ti} / \mathrm{Nb} / \mathrm{Al}, \mathrm{AlO}_{\mathrm{x}} / \mathrm{Al} / \mathrm{Nb} / \mathrm{NbN}$ STJs at $4.2 \mathrm{~K}(\mathrm{a}), 1.76 \mathrm{~K}$ (b), $1.25 \mathrm{~K}$ (c) and $0.1 \mathrm{~K}$ (d). 
trap is observed.

At temperatures below $\sim 2 \mathrm{~K}$, where the thermal quasiparticle current has decreased significantly, the I(V) curves show a sharp increase of the current at voltages above $\Delta / \mathrm{e}$ (Fig.1b and 2b). This contribution is caused by multiple-particle tunneling and Andreev reflection, is essentially independent of temperature, and depends on the uniformity of the insulating barrier [6].

At lower temperatures, A- and B-type STJs behave slightly differently in the voltage range between 0 and $\Delta / \mathrm{e}$ that is most important for STJ detector operation. In A-type STJs, the decrease in quasiparticle current with temperature follows the BCS model, with a typical hysteresis at $\mathrm{T}=1.25 \mathrm{~K}$ due to single quasiparticle tunneling in an asymmetric junction (Fig.1c). On the other hand, B-type STJ detectors with the Ti underlayer have no hysteresis at $\mathrm{T}=1.25 \mathrm{~K}$, and show a resistor-like linear current for $\mathrm{V}<\Delta / \mathrm{e}$ instead (Figure 2c). This linear current continues to decrease with temperature until $\mathrm{T} \approx 0.1 \mathrm{~K}$ (figure $2 \mathrm{~d}$, with peaks due to Fiske mode resonances that cannot be fully suppressed by the external magnetic field [7]).

One possible explanation for the observed quasiparticle currents in B-type STJs is the effect of the Ti-trap on the base electrode. In a simple model of $\mathrm{Nb} / \mathrm{Ti}$ base electrode, the superconducting gap near the tunnel barrier is determined mostly by the $\operatorname{Nb}$ layer $\left(\Delta_{\mathrm{Nb}} \approx 1.3\right.$ $\mathrm{meV})$, and the gap near the substrate is determined by the Ti-layer $\left(\Delta_{\mathrm{Ti}}=0.06 \mathrm{meV}\right)$. In this model the Ti layer cannot influence the tunneling current, as the current is determined by the quasiparticle density of states near the insulating barrier.

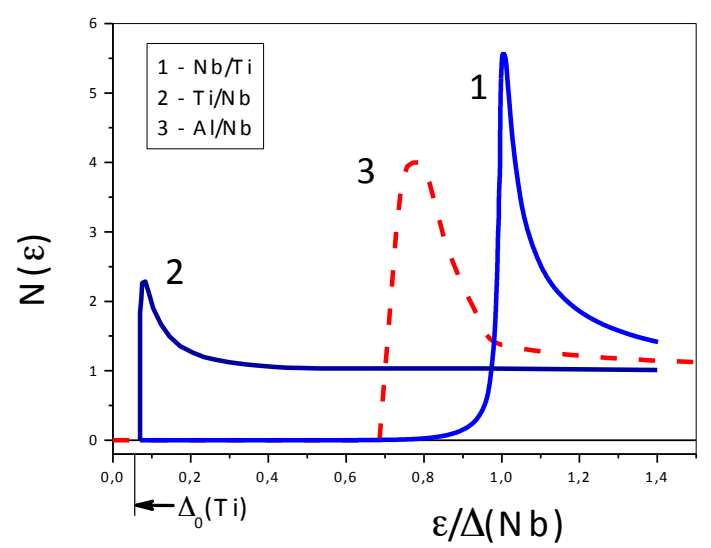

Fig. 3 (Color online) Density of quasiparticle states $N(\varepsilon, \mathrm{z})$ in the $\mathrm{Ti} / \mathrm{Nb} / \mathrm{Al}$ base electrode 1$)$ in Nb close to the tunnel barrier at $\mathrm{z}=0 ; 2$ ) at the bottom of the $\mathrm{Ti}$ film at $\mathrm{z}=\mathrm{d}_{\mathrm{Nb}}+\mathrm{d}_{\mathrm{Ti}} ; 3$ ) in the proximitized Al film.

However, a proper description of the $\mathrm{Nb} / \mathrm{Ti}$ base electrode should be based on the proximity theory in the dirty limit $[5,8]$. In this case the superconducting gap, i.e. the lowest possible energy for quasiparticle excitations, is constant for all layers in a multilayer electrode, while the density of states $N(\varepsilon, z)$ strongly depends on the coordinate $z$ perpendicular to the junction plane [8]. In the $\mathrm{Nb}$ film close to the tunnel barrier at $N(\varepsilon, z=0)$, the density of states (DOS) is mostly determined by $\mathrm{Nb}$, but there will 
be additional states at lower energies all the way down to the gap in the Ti trap, although the density of these low-energy states will be much lower.

Curve 1 in figure 3 shows the density of states calculated in [9] for a superconductor / normal metal sandwich at the outer side of the superconductor in the case of $d_{S} / \xi_{\mathrm{S}}=10$, where $d_{\mathrm{S}}$ is the thickness and $\xi_{\mathrm{S}}$ is the coherence length of the superconductor (Curve 7 in Fig.1b of [9]). At a qualitative level, these calculations are applicable to our case of the $\mathrm{Ti} / \mathrm{Nb} / \mathrm{Al}$ electrode near the tunnel barrier. The peak density of states at $\varepsilon=\Delta_{\mathrm{Nb}}$ corresponds to the gap in unperturbed $\mathrm{Nb}$. In addition, there is a tail in $N(\varepsilon)$ at lower energies due to the states in the $\mathrm{Ti}$ trap that extends down to the common superconducting energy gap. This low-energy tail in the density of states determines the tunneling current in STJs with a Ti base film at low temperatures. Curve 2 in figure 3 shows the expected density of states at the bottom of the Tilayer. Curve 3 shows $N(\varepsilon, \mathrm{z}=0)$ in the $\mathrm{Al}$ layer of the top $\mathrm{Al} / \mathrm{Nb} / \mathrm{NbN}$ electrode near the tunnel barrier.

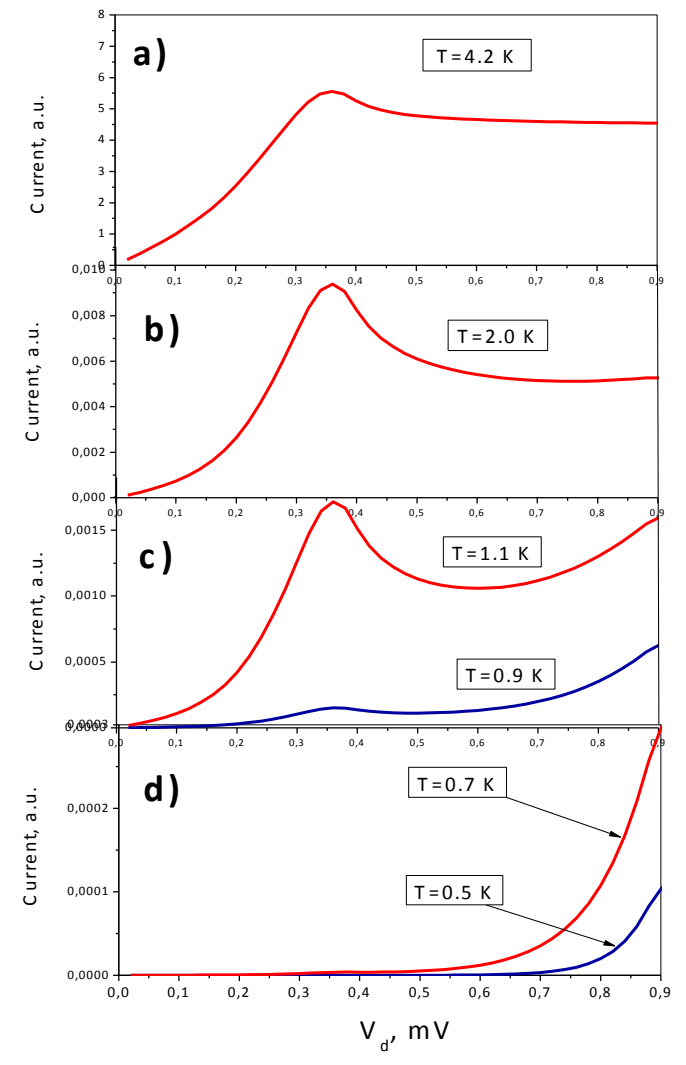

Fig. 4 (Color online) Calculated I(V) curves at various temperatures for the tunnel junction with $\Delta_{\text {top }}=0.96 \mathrm{meV}$ and $\Delta_{\text {killed }}=0.1 / 1.36 \mathrm{meV}$
Figure 4 shows the calculated I(V) curves at different temperatures, assuming densities of states according to figure 3 . In these calculations, the top electrode had an energy gap $\Delta_{\text {top }}=0.96 \mathrm{meV}$ (curve 3 in Fig. 3 ), and the base electrode had a maximum in the density of states at $\varepsilon=1.36 \mathrm{meV}$ and a tail in $N(\varepsilon)$ down to a common gap of $\Delta_{\text {base }}=$ $0.1 \mathrm{meV}$ (curve 1 in Fig. 3). Figure 4c shows a sharp decrease of the maximum current at $\mathrm{V}=0.4 \mathrm{meV}$ for temperatures between 1.1 and $0.9 \mathrm{~K}$. This causes the reduction of the hysteresis in the experimental I(V) curves. At lower temperatures (Fig. 4d), the calculated I(V) increases monotonically (Fig. 4d), similar to the experimental data at $\mathrm{T}=0.1 \mathrm{~K}$. Thus, our calculations agree with the experimental data on a qualitative level. 


\section{Conclusion}

While the addition of a low-gap film at the bottom of an STJ X-ray detector can remove the line splitting artifact and speed up the STJ response, it also introduces undesirable low-energy quasiparticle states at the tunnel barrier. This leads to a slower decrease of the quasiparticle current with temperature than expected from BCS theory alone, and can increase the electronic noise. In addition, quasiparticles captured in the low-gap film should have a nonzero probability for tunneling. This may slow down the detector response and lead to additional line broadening at low temperatures when the quasiparticle recombination in the low-gap layer becomes slow [10]. These effects can be reduced by using a thicker $\mathrm{Nb}$ layer in the base electrode with larger $\mathrm{d}_{\mathrm{Nb}} / \xi_{\mathrm{Nb}}$ [9]. They do not fundamentally preclude the fabrication of very fast STJ X-ray detectors with high energy resolution.

\section{Acknowledgements}

Part of this work was performed under the auspices of the U.S. Department of Energy by Lawrence Livermore National Laboratory under Contract DE-AC52-07NA27344.

\section{References}

1. P. Lerch, A. Zender, Quantum Giaever Detectors in Cryogenic Particle Detectors, vol. 99, ed. by C.Enss. Topics in Applied Physics (Springer, Berlin, 2005), pp. 217-265.

2. N. E. Booth, Appl. Phys. Lett. 50, 293 (1987).

3. O.J. Luiten, M.L. Van den Berg, J. Gomez Rivas, M.P. Bruijn, F.B. Kiewiet, P.A.J. de Korte, in Proc. of 7th Intern. Workshop on Low Temperature Detectors, ed. by S. Cooper. Munich (1997), p. 25.

4. V. A. Andrianov, LV. Filippenko, V.P. Gorkov, V.P. Koshelets, Nucl. Instr. And Methods A 559, 683 (2006).

5. A.A. Golubov, E. P. Houwman, J. G. Gijsbertsen, V. M. Krasnov, J. Flokstra, H. Rogalla, M. Y. Kupriyanov. Phys. Rev. B. 51, 1073 (1995).

6. C.L. Foden, N. Rando, A. van Dordrecht, A. Peacock, J. Lumley, C. Pereira. Phys. Rev. B. 47, 3316 (1993).

7. S. Friedrich, M.F. Cunningham, M. Frank, S.E. Labov, A.T. Barfknecht, S.P. Cramer. Nucl. Instr. Meth. A 444, $151(2000)$.

8. G. Brammertz, A. Poelaert, A. A. Golubov, P. Verhoeve, A. Peacock, H. Rogalla. J. Appl. Phys. 90, 355 (2001)

9. A.A. Golubov and M.Y. Kupriyanov. Russian Microelectronics 14, № 5, 213 (1985).

10. V.A. Andrianov, M.G. Kozin, P.N. Dmitriev, V.P. Koshelets, I.L. Romashkina, S.A. Sergeev, AIP Conf. Proc. 605, 161 (2002). 